\title{
Optimal Design of Vector Smoothing Spline Curves with Constraints
}

\author{
Hiroyuki Fujioka ${ }^{1)}$ and Hiroyuki Kano ${ }^{2)}$ \\ 1) Department of System Management, Fukuoka Institute of Technology \\ 3-30-1, Wajiro-higashi, Higashi-ku, Fukuoka 811-0295, JAPAN \\ E-mail: fujioka@fit.ac.jp \\ 2) Division of Science, School of Science and Engineering, Tokyo Denki University \\ Hatoyama, Hiki-gun, Saitama 350-0394, JAPAN \\ E-mail: kano@mail.dendai.ac.jp
}

\begin{abstract}
This paper considers the problem for designing of optimal vector smoothing splines with equality and/or inequality constraints. The vector splines are constituted employing normalized uniform B-splines as the basis functions. Then various types of constraints are formulated as linear function of the so-called control points, and the problem is reduced to quadratic programming problem. The performance is examined by some numerical examples.
\end{abstract}

\section{Introduction}

In many applications of science and engineering, the problems of constructing curves for a given set of discrete observational data, arise. For such problems, a commonly-used way is to use interpolating and approximating methods using spline functions and they have been studied extensively (e.g. [1, 2, 3]).

One of the advantages of using spline functions is its computational feasibility. In particular, using B-splines as the basis functions $[3,4]$ yields simple algorithms for designing curves, and moreover enables us to extend the results for the one-dimensional case (i.e. the case of curves) to the two-dimensional case (i.e. the case of surfaces) [5] and to even higher dimensions.

In addition to traditional approximating or interpolating splines, there are a large class of problems where we need to impose various constraints on splines such as monotone smoothing splines [6], inequality constraints at isolated points [7], etc. Employing B-splines approach, the authors have developed a method for designing smoothing splines with constraints over interval or at isolated points, and the construction of the spline then becomes a quadratic programming problem [8].

This paper is a continuation of our studies on the optimal design of constrained spline curves based on B-spline approach in [8]. We here generalize the design method so that the multiple curves can be designed simultaneously with equality and/or inequality constraints. In addition, we newly consider a type of interval constraints specified by some functions. Em- ploying the idea of limiting spline curves [5], it then enables us to ensure that smoothing spline curves obey such constraints over interval. Such a design method may be useful in many applications, e.g. estimation of probability density functions [9] and trajectory planning for robotic motions [10], etc. The performance is examined by some numerical examples.

This paper is organized as follows. In Section 2, we briefly review B-splines and design methods of optimal vector splines. Then in Section 3, we show how various types of constraints on splines can be formulated and solved. We examine the performances of the proposed method by numerical examples in Section 4. Concluding remarks are given in Section 5 .

We summarize some of the symbols that will be used throughout the paper: $\otimes$ the Kronecker product, and 'vec' denotes the vec-function, i.e. for a matrix $A=\left[\begin{array}{llll}a_{1} & a_{2} & \cdots & a_{n}\end{array}\right] \in \mathbf{R}^{m \times n}$ with $a_{i} \in \mathbb{R}^{m}$, vec $A=\left[\begin{array}{llll}a_{1}^{T} & a_{2}^{T} & \cdots & a_{n}^{T}\end{array}\right]^{T} \in \mathbf{R}^{m n}$ (see e.g. [11]).

\section{Optimal Smoothing Splines}

Let $x(t) \in \mathbf{R}^{p}(p \geq 1)$ be vector spline curves defined by

$$
x(t)=\left[\begin{array}{llll}
x_{1}(t) & x_{2}(t) & \cdots & x_{p}(t)
\end{array}\right]^{T} .
$$

Then arbitrary vector spline curves $x(t)$ of degree $k$ in an interval $\mathcal{D}=\left[t_{0}, t_{m}\right] \subset \mathbf{R}$ can be represented as

$$
x(t)=\sum_{i=-k}^{m-1} \tau_{i} B_{k}\left(\alpha\left(t-t_{i}\right)\right),
$$

by an appropriate choice of the weighting coefficient vector $\tau_{i}=\left[\tau_{1, i}, \tau_{2, i}, \cdots, \tau_{p, i}\right]^{T} \in \mathbf{R}^{p}$ called control points [3]. Here, $B_{k}(t)$ is a normalized, uniform Bspline function of degree $k, m$ is an integer, and $\alpha(>0)$ is a constant for scaling the interval between equallyspaced knot points $t_{i}$ with

$$
t_{i+1}-t_{i}=\frac{1}{\alpha}
$$

It is noted that employing a higher degree $k$ of B-splines in (2) yields splines $x(t)$ of higher degree and thus allows 
us to design more complex curves. Also, for fixed $k$ and the interval $\left[t_{0}, t_{m}\right]$, increasing the parameter $\alpha$ (i.e. smaller knot points spacing) gives us more flexibility of spline design since $m$ (equivalently the number of control points) increases.

\subsection{Normalized Uniform B-Splines}

Normalized uniform B-spline $B_{k}(t)$ of degree $k$ is defined by

$$
B_{k}(t)= \begin{cases}N_{k-j, k}(t-j) & j \leq t<j+1, \\ & j=0,1, \cdots, k \\ 0 & t<0 \text { or } t \geq k+1,\end{cases}
$$

and the basis elements $N_{j, k}(t)(j=0,1, \cdots, k), 0 \leq t \leq$ 1 are obtained recursively by the following algorithm:

Algorithm 1 Let $N_{0,0}(t) \equiv 1$ and, for $i=1,2, \cdots, k$, compute

$$
\left\{\begin{aligned}
N_{0, i}(t) & =\frac{1-t}{i} N_{0, i-1}(t) \\
N_{j, i}(t) & =\frac{i-j+t}{i} N_{j-1, i-1}(t)+\frac{1+j-t}{i} N_{j, i-1}(t), \\
& \quad j=1, \cdots, i-1 \\
N_{i, i}(t) & =\frac{t}{i} N_{i-1, i-1}(t) .
\end{aligned}\right.
$$

Thus, $B_{k}(t)$ is a piece-wise polynomial of degree $k$ with integer knot points and is $k-1$ times continuously differentiable. It is noted that $B_{k}(t)$ for $k=0,1,2, \cdots$ is normalized in the following sense

$$
\sum_{j=0}^{k} N_{j, k}(t)=1, \quad 0 \leq t \leq 1,
$$

and this yields

$$
\int_{-\infty}^{\infty} B_{k}(t) d t=\int_{0}^{k+1} B_{k}(t) d t=1 .
$$

For the sake of later reference, we introduce $(k+1)$ dimensional vectors $N_{k}(t)$ and $h_{k}(t)$ as

$$
\begin{aligned}
N_{k}(t) & =\left[\begin{array}{llll}
N_{0, k}(t) & N_{1, k}(t) & \cdots & N_{k, k}(t)
\end{array}\right]^{T}(8) \\
h_{k}(t) & =\left[\begin{array}{llll}
t^{k} & t^{k-1} & \cdots & 1
\end{array}\right]^{T} .
\end{aligned}
$$

Then $N_{k}(t)$ is written as

$$
N_{k}(t)=S_{k} h_{k}(t),
$$

where $S_{k} \in \mathbf{R}^{(k+1) \times(k+1)}$ is a matrix whose $i$-th row consists of the coefficients of polynomial $N_{i-1, k}(t)$. It can be shown that the matrix $S_{k}$ can be obtained by the following recursive algorithm. Letting $S_{0}=1$, compute $S_{i} \in \mathbf{R}^{(i+1) \times(i+1)}$ for $i=1,2, \cdots, k$ by

$$
S_{i}=\frac{1}{i}\left(\left[\begin{array}{ll}
0_{i+1} & \Gamma_{i} S_{i-1}
\end{array}\right]+\left[\begin{array}{ll}
\Delta_{i} S_{i-1} & 0_{i+1}
\end{array}\right]\right),
$$

where the matrices $\Gamma_{i}, \Delta_{i} \in \mathbf{R}^{(i+1) \times i}$ are defined as

$$
\Gamma_{i}=\left[\begin{array}{cccccc}
1 & & & & & \\
i-1 & 2 & & & & \\
& i-2 & 3 & & & \\
& & \ddots & \ddots & & \\
& & & \ddots & \ddots & \\
& & & & 1 & i \\
& & & & 0 & 0
\end{array}\right] \text {, }
$$

and

$$
\Delta_{i}=\left[\begin{array}{cccccc}
-1 & & & & & \\
1 & -1 & & & & \\
& 1 & -1 & & & \\
& & \ddots & \ddots & & \\
& & & \ddots & \ddots & \\
& & & & 1 & -1 \\
& & & & & 1
\end{array}\right] .
$$

Here the empty spaces denote zero entries. When $k=$ 3 , for example, we obtain the matrix $S_{3}$ as

$$
S_{3}=\frac{1}{3 !}\left[\begin{array}{cccc}
-1 & 3 & -3 & 1 \\
3 & -6 & 0 & 4 \\
-3 & 3 & 3 & 1 \\
1 & 0 & 0 & 0
\end{array}\right]
$$

\subsection{Optimal Vector Smoothing Splines}

The control point vector $\tau_{i} \in \mathbf{R}^{p}(p \geq 1)$ in (2) are typically determined by the theory of smoothing splines (see, e.g. [4] for details). Suppose that we are given a set of data

$$
\left\{\left(s_{i}, d_{i}\right): s_{i} \in\left[t_{0}, t_{m}\right], d_{i} \in \mathbf{R}^{p}, i=1,2, \cdots, N\right\},
$$

and let $\tau \in \mathbf{R}^{p \times M}(M=m+k)$ be the weight matrix defined by

$$
\tau=\left[\begin{array}{cccc}
\tau_{1,-k} & \tau_{1,-k+1} & \cdots & \tau_{1, m-1} \\
\tau_{2,-k} & \tau_{2,-k+1} & \cdots & \tau_{2, m-1} \\
\vdots & \vdots & \vdots & \vdots \\
\tau_{p,-k} & \tau_{p,-k+1} & \cdots & \tau_{p, m-1}
\end{array}\right] .
$$

Then a standard problem is to find such a $\tau$ minimizing the cost function

$$
J(\tau)=\int_{t_{0}}^{t_{m}}\left\|x^{(2)}(t)\right\|_{\Lambda}^{2} d t+\sum_{i=1}^{N}\left\|x\left(s_{i}\right)-d_{i}\right\|_{W_{i}}^{2},
$$

where $\|z\|_{S}^{2}=z^{T} S z, \Lambda=\operatorname{diag}\left\{\lambda_{1}, \lambda_{2}, \ldots, \lambda_{p}\right\} \in \mathbf{R}^{p \times p}$ with smoothing parameter $\lambda_{i}(>0), \forall i$, and $W_{i}=W_{i}^{T} \in$ $\mathbf{R}^{p \times p}$ satisfies $I_{3} \geq W_{i} \geq 0, \forall i$.

Letting $\hat{\tau} \in \mathbf{R}^{p M}$ be vec-function of $\tau \in \mathbf{R}^{p \times M}$, i.e.

$$
\hat{\tau}=\operatorname{vec} \tau,
$$

the cost function $J(\tau)$ can be rewritten as a quadratic function $J(\hat{\tau})$ in terms of $\tau$,

$$
J(\hat{\tau})=\hat{\tau}^{T} G_{N} \hat{\tau}-2 \hat{\tau}^{T} g_{N}+\text { const. }
$$


with

$$
\begin{aligned}
G_{N} & =Q \otimes \Lambda+\sum_{i=1}^{N}\left(b\left(s_{i}\right) b^{T}\left(s_{i}\right)\right) \otimes W_{i} \\
g_{N} & =\sum_{i=1}^{N} b\left(s_{i}\right) \otimes W_{i} d_{i} .
\end{aligned}
$$

Here $Q \in \mathbf{R}^{M \times M}$ is a Gramian defined by

$$
Q=\int_{t_{0}}^{t_{m}} \frac{d^{2} b(t)}{d t^{2}} \frac{d^{2} b^{T}(t)}{d t^{2}} d t
$$

with

$$
\begin{aligned}
& b(t)=\left[\begin{array}{lll}
B_{k}\left(\alpha\left(t-t_{-k}\right)\right) & B_{k}\left(\alpha\left(t-t_{-k+1}\right)\right)
\end{array}\right. \\
& \left.\cdots B_{k}\left(\alpha\left(t-t_{m-1}\right)\right)\right]^{T} .
\end{aligned}
$$

Obviously, if there are no constraints, the optimal solution is given as a solution of linear algebraic equations,

$$
G_{N} \hat{\tau}=g_{N},
$$

On the other hand, a given function $f(t)=$ $\left[f_{1}(t), f_{2}(t), \cdots, f_{p}(t)\right]^{T} \in \mathbb{R}^{p}, t \in\left[t_{0}, t_{m}\right]$ can also be approximated by smoothing splines, in which case the following cost function is used.

$$
J(\tau)=\int_{t_{0}}^{t_{m}}\left\|x^{(2)}(t)\right\|_{\Lambda}^{2} d t+\int_{t_{0}}^{t_{m}}\|x(t)-f(t)\|^{2} .
$$

Similarly as above, this cost function is rewritten as

$$
J(\hat{\tau})=\hat{\tau}^{T} G_{c} \hat{\tau}-2 \hat{\tau}^{T} g_{c}+f_{0}
$$

where

$$
\begin{aligned}
G_{c} & =Q \otimes \Lambda+\int_{t_{0}}^{t_{m}} b(t) b^{T}(t) \otimes I_{p} d t \\
g_{c} & =\int_{t_{0}}^{t_{m}} b(t) \otimes f(t) d t \\
f_{0} & =\int_{t_{0}}^{t_{m}}\|f(t)\|^{2} d t .
\end{aligned}
$$

Let $x^{c}(t)=\left[x_{1}^{c}, x_{2}^{c}, \cdots, x_{p}^{c}\right]^{T} \in \mathbf{R}^{p}$ and $\tau^{c}=$ $\left[\tau_{-k}^{c}, \tau_{-k+1}^{c}, \cdots, \tau_{m-1}^{c}\right] \in \mathbf{R}^{p \times M}$ be the approximating curves of the above problem and the corresponding control point matrix, where $\tau_{i}^{c}=\left[\tau_{1, i}^{c}, \tau_{2, i}^{c}, \cdots, \tau_{p, i}^{c}\right]^{T} \in$ $\mathbf{R}^{p}$. Then if the data $d_{i}$ in (15) for constructing smoothing curves are obtained by sampling $f(t)$, it holds under certain natural conditions to $x^{c}(t)$ as the number of data $N$ increases (see [5] for details). In the sequence, such approximating spline curves $x^{c}(t)$ are referred to as "limiting splines".

\section{Optimal Splines with Constraints}

There are various types of constraints on splines $x(t), t \in\left[t_{0}, t_{m}\right]$, e.g. those for $x(t)$ and/or its derivatives, for some $t$ or for all $t$, for equality and/or inequality, etc. Here we first develop basic formula for expressing the constraints.
Since each element of $x(t)$ in (1), i.e. $x_{q}(t), q=$ $1,2, \cdots, p$, is a piece-wise polynomial of degree $k$, we examine the polynomial in each interval $\left[t_{j}, t_{j+1}\right)$ for $j=0,1, \cdots, m-1$. By focusing on the interval $\left[t_{j}, t_{j+1}\right), x_{q}(t)$ is written as

$$
x_{q}(t)=\sum_{i=-k+j}^{j} \tau_{q, i} B_{k}\left(\alpha\left(t-t_{i}\right)\right) .
$$

Using (4), we then get

$$
x_{q}(t)=\sum_{i=0}^{k} \tau_{q, j-k+i} N_{i, k}\left(\alpha\left(t-t_{j}\right)\right), \quad t \in\left[t_{j}, t_{j+1}\right),
$$

and it depends on only the $k+1$ weights $\tau_{q, j-k}, \tau_{q, j-k+1}$, $\cdots, \tau_{q, j}$. Moreover, by introducing a new variable $u$,

$$
u=\alpha\left(t-t_{j}\right),
$$

the interval $\left[t_{j}, t_{j+1}\right)$ in $t$ is normalized to $[0,1)$ in $u$, and we may write $x_{q}(t)$ as $\hat{x}_{q}(u)$,

$$
\hat{x}_{q}(u)=\sum_{i=0}^{k} \tau_{q, j-k+i} N_{i, k}(u), \quad u \in[0,1) .
$$

Letting $\tau_{(j)}^{q}=\left[\tau_{q, j-k}, \tau_{q, j-k+1}, \cdots, \tau_{q, j}\right]^{T} \in \mathbf{R}^{k+1}$ and using (10), we may rewrite $\hat{x}_{q}(u)$ in (33) as $\hat{x}_{q}(u)=$ $N_{k}^{T}(u) \tau_{(j)}^{q}$ and hence $x_{q}(t)=N_{k}^{T}(u) \tau_{(j)}^{q}$.

In general, the $l$-th derivative $x_{q}^{(l)}(t)$ for $t \in\left[t_{j}, t_{j+1}\right)$ is expressed in terms of $u \in[0,1)$ in (32) by

$$
x_{q}^{(l)}(t)=\alpha^{l} \hat{x}_{q}^{(l)}(u), l=0,1,2, \cdots,
$$

with

$$
\hat{x}_{q}^{(l)}(u)=\left(N_{k}^{(l)}(u)\right)^{T} \tau_{(j)}^{q} .
$$

Here $N_{k}^{(l)}(u)$ is obtained from (10) as

$$
N_{k}^{(l)}(u)=S_{k} C_{k, l} h_{k-l}(u),
$$

where $C_{k, l} \in \mathbf{R}^{(k+1) \times(k-(l-1))}$ is defined by

$$
C_{k, l}=C_{k} C_{k-1} \cdots C_{k-(l-1)},
$$

and $C_{i} \in \mathbf{R}^{(i+1) \times i}$ by

$$
C_{i}=\left[\begin{array}{cccc}
i & & & \\
& i-1 & & \\
& & \ddots & \\
& & & 1 \\
0 & \cdots & \cdots & 0
\end{array}\right] .
$$

Here the empty spaces denote zero entries.

Now we are in a position to derive various types of constraints on $x(t)$. For the sake of simplicity, we consider the case of cubic splines, i.e. $k=3$. 


\subsection{Pointwise Constraints}

By (34) and (35), we observe that any constraint on the value of $x_{q}^{(l)}(t), q=1,2, \cdots, p$, for given $t \in$ $\left[t_{j}, t_{j+1}\right)$ is specified as a linear constraint on the vector $\hat{\tau} \in \mathbb{R}^{p M}$, since $\tau_{(j)}^{q} \in \mathbb{R}^{4}$ is a sub-vector of $\hat{\tau}$. Specifically, $x_{q}^{(l)}(t)$ is written as

$$
x_{q}^{(l)}(t)=\left(a \otimes v_{q}\right)^{T} \hat{\tau}
$$

where

$$
\begin{aligned}
a^{T} & =\left[\begin{array}{lll}
0_{j}^{T} & \alpha^{l} N_{3}^{(l)}(u)^{T} & 0_{M-j-4}^{T}
\end{array}\right], \\
v_{q}^{T} & =\left[\begin{array}{lll}
0_{q-1}^{T} & 1 & 0_{p-q-1}^{T}
\end{array}\right] .
\end{aligned}
$$

Only the point in $\left[t_{0}, t_{m}\right]$ that is not covered in the foregoing arguments is $t=t_{m}$. However, the values of $x_{q}^{(l)}(t)$ at $t=t_{m}$ for $l=0,1,2$ are obtained, by the continuity of these functions. Namely, by letting $j=m-1$ and $u=1$, we have the expression $x_{q}^{(l)}\left(t_{m}\right)=$ $\left(a \otimes v_{q}\right)^{T} \hat{\tau}$ with

$$
a^{T}=\left[\begin{array}{ll}
0_{M-4}^{T} & \alpha^{l} N_{3}^{(l)}(1)^{T}
\end{array}\right] .
$$

If we need to constrain $x_{q}^{(3)}(t)$ at $t=t_{m}$, which is piecewise constant and is discontinuous at the knot points, we simply regard that $x_{q}^{(3)}\left(t_{m}\right)=\lim _{t \rightarrow t_{m}} x_{q}^{(3)}(t)$, implying that (42) holds also for $l=3$.

When we impose the above constraints for all the $x_{q}(t)$ simultaneously, i.e. $x^{(l)}(t), t \in\left[t_{j}, t_{j+1}\right]$, the constraints corresponding to (39) can be written as

$$
x^{(l)}(t)=\left[\begin{array}{c}
\left(a \otimes v_{1}\right)^{T} \\
\left(a \otimes v_{2}\right)^{T} \\
\vdots \\
\left(a \otimes v_{p}\right)^{T}
\end{array}\right] \hat{\tau}=\left(a \otimes I_{p}\right)^{T} \hat{\tau} .
$$

In particular, if $t$ is taken to be the knot point $t=$ $t_{j}(j=0,1, \cdots, m-1)$ and hence $u=0$, we obtain $N_{3}^{(l)}(0)^{T}$ from (36) as

$$
N_{3}^{(l)}(0)^{T}= \begin{cases}\frac{1}{6}\left[\begin{array}{llll}
1 & 4 & 1 & 0
\end{array}\right] & l=0 \\
\frac{1}{2}\left[\begin{array}{llll}
-1 & 0 & 1 & 0
\end{array}\right] & l=1 \\
{\left[\begin{array}{llll}
1 & -2 & 1 & 0
\end{array}\right]} & l=2 \\
{\left[\begin{array}{llll}
-1 & 3 & -3 & 1
\end{array}\right]} & l=3\end{cases}
$$

For example, in order to impose the initial condition $x\left(t_{0}\right)=x^{(1)}\left(t_{0}\right)=x^{(2)}\left(t_{0}\right)=0_{p}$, we simply introduce the condition $\left(A \otimes I_{p}\right)^{T} \hat{\tau}=0_{3 p}$, where $A^{T} \in \mathrm{R}^{3 \times M}$ is derived from (40) with $j=0$ and (44) as

$$
\begin{aligned}
A^{T} & =\left[\begin{array}{cc}
N_{3}^{(0)}(0)^{T} & 0_{M-4}^{T} \\
\alpha N_{3}^{(1)}(0)^{T} & 0_{M-4}^{T} \\
\alpha^{2} N_{3}^{(2)}(0)^{T} & 0_{M-4}^{T}
\end{array}\right] \\
= & {\left[\begin{array}{cccccc}
\frac{1}{6} & \frac{4}{6} & \frac{1}{6} & 0 & \cdots & 0 \\
-\frac{\alpha}{2} & 0 & \frac{\alpha}{2} & 0 & \cdots & 0 \\
\alpha^{2} & -2 \alpha^{2} & \alpha^{2} & 0 & \cdots & 0
\end{array}\right] }
\end{aligned}
$$

Terminal conditions for $t=t_{m}$ may be defined similarly by using (43).

To conclude, this method can be used to specify equality or inequality constraint on $x(t)$ or its element $x_{q}^{(l)}(t), q=1,2, \cdots, p$, for given $t \in\left[t_{0}, t_{m}\right)$ and $l=0,1,2, \cdots, k$ as linear constraint on $\hat{\tau}$.

\subsection{Constraints over Knot Point Intervals}

Next we consider the cases of constraints over knot point intervals. Specifically, we consider an inequality constraint as

$$
x_{q}(t) \geq z_{q}(t) \quad \forall t \in\left[t_{j}, t_{j+1}\right]
$$

for a given continuous function $z_{q}(t), q=1,2, \cdots, p$ of $z(t)=\left[z_{1}(t), z_{2}(t), \cdots, z_{p}(t)\right]^{T} \in \mathbb{R}^{p}$. Note that this inequality ' $\geq$ may readily be replaced with ${ }^{\prime} \leq$ and equality ${ }^{\prime}={ }^{\prime}$.

The constraint in (46) may be imposed by employing the idea of "limiting splines" in (25) as follows : For the given function $z_{q}(t)$, we compute the limiting splines $x_{q}^{c}(t)$ with the same form and the same degree $k=3$ as in (2), i.e.

$$
x_{q}^{c}(t)=\sum_{i=-3}^{m-1} \tau_{q, i}^{c} B_{3}\left(\alpha\left(t-t_{i}\right)\right) .
$$

From our past works (e.g. see [5]), we have empirically confirmed that the curves $x_{q}^{c}(t)$ can approximate functions $z_{q}(t)$ fairly precisely, i.e. $x_{q}^{c}(t) \approx z_{q}(t)$. We thus use the constraint $x_{q}(t) \geq x_{q}^{c}(t)$ instead of $x_{q}(t) \geq z_{q}(t)$. Then, the constraints may be realized by imposing the condition $\tau_{q, i} \geq \tau_{q, i}^{c}$ for $i=j-3, j-2, j-1, j$, or in terms of vectors $\hat{\tau}$ and $\hat{\tau}_{c}$ as

$$
\left(E_{j} \otimes v_{q}\right)^{T} \hat{\tau} \geq\left(E_{j} \otimes v_{q}\right)^{T} \hat{\tau}_{c},
$$

where $\hat{\tau}^{c}=\operatorname{vec} \tau^{c} \in \mathbf{R}^{p M}$ and $E_{j}^{T} \in \mathbf{R}^{4 \times M}$ is defined by

$$
E_{j}^{T}=\left[\begin{array}{lll}
0_{4, j} & I_{4} & 0_{4, M-j-4}
\end{array}\right] .
$$

This is because, if $\tau_{q, i} \geq \tau_{q, i}^{c}$ holds, we have from (31)(33),

$$
\begin{gathered}
x_{q}(t)=\hat{x}_{q}(u)=\sum_{i=0}^{3} \tau_{q, j-3+i} N_{i, 3}(u) \\
\geq \sum_{i=0}^{3} \tau_{q, j-3+i}^{c} N_{i, 3}(u)=\hat{x}_{q}^{c}(u)=x_{q}^{c}(t) \\
\forall t \in\left[t_{j}, t_{j+1}\right),(50)
\end{gathered}
$$

since $N_{i, 3}(u) \geq 0 \forall u \in[0,1]$.

The above arguments are easily extended to larger knot point interval $\left[t_{j}, t_{l}\right]$ for some $l(>j)$ : The constraint

$$
x_{q}(t) \geq x_{q}^{c}(t) \quad \forall t \in\left[t_{j}, t_{l}\right]
$$

is satisfied by the condition $\tau_{q, i} \geq \tau_{q, i}^{c}, \quad i=j-3, j-$ $2, \cdots, l-1$, or equivalently

$$
\left(E_{j, l} \otimes v_{q}\right)^{T} \hat{\tau} \geq\left(E_{j, l} \otimes v_{q}\right)^{T} \hat{\tau}^{c},
$$


where $E_{j, l}^{T} \in \mathbf{R}^{(l-j+3) \times M}$ is defined by

$$
E_{j, l}^{T}=\left[\begin{array}{lll}
0_{l-j+3, j} & I_{l-j+3} & 0_{l-j+3, M-l-3}
\end{array}\right] .
$$

A special case of the above may be $z_{q}(t)=c$ in (46) with a constant $c$. Then, the condition corresponding to (48), i.e. $x_{q}(t) \geq c \forall t \in\left[t_{j}, t_{j+1}\right]$ may be simplified without using the limiting splines $x_{q}^{c}(t)$ as

$$
\left(E_{j} \otimes v_{q}\right)^{T} \hat{\tau} \geq \mathrm{c}_{4},
$$

where $\mathbf{c}_{4}$ denotes $\mathbf{c}_{i}=(c c \cdots c)^{T} \in \mathbf{R}^{i}$ in general. In fact, conditions (54) and (6) yield

$$
\begin{array}{r}
x_{q}(t)=\hat{x}_{q}(u)=\sum_{i=0}^{3} \tau_{q, j-3+i} N_{i, 3}(u) \\
\geq \sum_{i=0}^{3} c N_{i, 3}(u)=c \sum_{i=0}^{3} N_{i, 3}(u)=c \\
\forall t \in\left[t_{j}, t_{j+1}\right) .
\end{array}
$$

The knot point interval $\left[t_{j}, t_{j+1}\right]$ is extended to arbitrary length $\left[t_{j}, t_{l}\right](l>j)$ as

$$
\left(E_{j, l} \otimes v_{q}\right)^{T} \hat{\tau} \geq \mathrm{c}_{l-j+3} .
$$

It is noted that the conditions in (48) are only sufficient for $x_{q}(t) \geq x_{q}^{c}(t) \forall t \in\left[t_{j}, t_{j+1}\right]$, and the same is true for (52), (54) and (56).

When the above constraints are imposed for all the elements $x_{q}(t)$ simultaneously, i.e. $x(t) \geq x^{c}(t)(\approx z(t))$ and $x(t) \geq \mathbf{c}_{p}$, we have only to replace $v_{q}$ with $I_{p}$ in (48), (52), (54) and (56).

\subsection{Constraints on Integral Values}

We finally consider the case for an equality or inequality constraint on the value of integral $\int_{t_{0}}^{t_{m}} x_{q}(t) d t$. From (34), (35) with $l=0$ and (32), we get

$$
\begin{aligned}
\int_{t_{0}}^{t_{m}} x_{q}(t) d t & =\sum_{j=0}^{m-1} \int_{t_{j}}^{t_{j+1}} x_{q}(t) d t \\
& =\frac{1}{\alpha} \sum_{j=0}^{m-1} \int_{0}^{1} \hat{x}_{q}(u) d u \\
& =\frac{1}{\alpha} \sum_{j=0}^{m-1} \tau_{(j)}^{q} \int_{0}^{1} N_{3}^{T}(u) d u
\end{aligned}
$$

$\begin{array}{lrcr}\text { Noting } & \text { that } & N_{3}^{T}(u) & = \\ {\left[N_{0,3}(u),\right.} & N_{1,3}(u), & N_{2,3}(u), & \left.N_{3,3}(u)\right]\end{array}$ and $\tau_{(j)}^{q}=\left[\tau_{q, j-3}, \tau_{q, j-2}, \tau_{q, j-1}, \tau_{q, j}\right]^{T}$, we obtain

$$
\begin{aligned}
\alpha \int_{t_{0}}^{t_{m}} x_{q}(t) d t & =\sum_{j=-3}^{-1} \tau_{q, j} \sum_{i=0}^{j+3} \int_{0}^{1} N_{i, 3}(u) d u \\
& +\sum_{j=0}^{m-4} \tau_{q, j} \sum_{i=0}^{3} \int_{0}^{1} N_{i, 3}(u) d u \\
& +\sum_{j=m-3}^{m-1} \tau_{q, j} \sum_{i=j-m+4}^{3} \int_{0}^{1} N_{i, 3}(u) d u
\end{aligned}
$$

Here, using (10) and (9), we get

$$
\int_{0}^{1} N_{3}(u) d u=\frac{1}{24}\left[\begin{array}{llll}
1 & 11 & 11 & 1
\end{array}\right]^{T} .
$$

From the above two equations, it can be shown that the integral value $\int_{t_{0}}^{t_{m}} x_{q}(t) d t$ is expressed as a linear function in $\hat{\tau}$,

$$
\int_{t_{0}}^{t_{m}} x_{q}(t) d t=\left(a \otimes v_{q}\right)^{T} \hat{\tau}
$$

where $a \in \mathbf{R}^{M}$ is given by

$$
a=\frac{1}{24 \alpha}\left[\begin{array}{lllllllll}
1 & 12 & 23 & 24 & \cdots & 24 & 23 & 12 & 1
\end{array}\right]^{T} \text {. }
$$

Replacing $v_{q}$ in (58) with $I_{p}$, the integral value $\int_{t_{0}}^{t_{m}} x(t) d t$ can be obtained as a linear function in $\hat{\tau}$, hence $\int_{t_{0}}^{t_{m}} x(t) d t=\left(a \otimes I_{p}\right)^{T} \hat{\tau}$.

\subsection{Constrained Splines}

As the foregoing development indicates, we can expect that a fairly large number of constrained spline problems may be treated in the above settings. The formulation is simple and is very well fit for numerical solutions as quadratic programing problems. Namely, the optimal smoothing or interpolating splines are obtained by minimizing the quadratic cost $J(\hat{\tau})$ as shown in (19) and (26), whereas a number of constraints on the splines may be expressed as linear constraints on $\hat{\tau}$, either equality or inequality or both. A general form of problems is

$$
\min _{\tau \in \mathbb{R}^{M}} J(\hat{\tau})=\frac{1}{2} \hat{\tau}^{T} G \hat{\tau}+g^{T} \hat{\tau}
$$

subject to the constraints of the form

$$
A \hat{\tau}=d, f_{1} \leq E \hat{\tau} \leq f_{2}, h_{1} \leq \hat{\tau} \leq h_{2},
$$

for some matrices and vectors of appropriate dimensions. A very efficient numerical algorithm is available for this purpose.

On the other hand, if only equality constraints are to be imposed on the spline, we could clearly proceed further by using Lagrange function,

$$
L(\hat{\tau}, \mu)=J(\hat{\tau})+\mu^{T}(A \hat{\tau}-d) .
$$

By the standard procedure, we get a system of equations in $\hat{\tau}$ and the Lagrange multipliers $\mu$,

$$
\left[\begin{array}{cc}
G & A^{T} \\
A & 0
\end{array}\right]\left[\begin{array}{l}
\hat{\tau} \\
\mu
\end{array}\right]=\left[\begin{array}{c}
-g \\
d
\end{array}\right]
$$

This is the case when we specify the initial and/or terminal conditions on $x(t)$ or its elements as described in Section 3.1. For example, periodic splines can also be treated in these settings by imposing $\tau_{j}=\tau_{m+j}, j=$ $-k,-k+1, \cdots,-1$, which yields $x^{(i)}\left(t_{0}\right)=x^{(i)}\left(t_{m}\right), i=$ $0,1, \cdots, k-1$ and hence the periodicity, 


\section{Numerical Examples}

We examine the design method presented in the previous sections numerically. In all the cases, cubic splines, i.e. $k=3$, is used.

\subsection{Approximation of Probability Density Functions}

We approximate a probability density function from the histogram of random samples for the case of $p=1$. Figure 1 shows the histogram of $100\left(=N_{s}\right)$ Gaussian random numbers with zero mean and unit standard deviation. We approximate the density function in the interval $\left[t_{0}, t_{m}\right]=[-5,+5]$. The data $\left(s_{i}, d_{i}\right), i=$ $1,2, \cdots, N$ in (15) is then generated as follows. First, $s_{i}$ 's are taken as the center of each bins in the histogram as $s_{1}=-5, s_{2}=-4, \cdots, s_{11}=5$, and hence the number of data is $N=11$. The data $d_{i}$ is then obtained by rescaling the histogram, say $H_{i}, i=1,2, \cdots, 11$, so that the area covered by the histogram over $\left[t_{0}, t_{m}\right]$ is normalized to one, yielding $d_{i}=H_{i} / 100$. Note that, in general, the histogram is scaled as $d_{i}=H_{i} / S$ with $S=N_{s} \frac{t_{m}-t_{0}}{N-1}$, and the pair $\left(s_{i}, d_{i}\right)$ can be used for reconstructing probability density functions.

With $k=3, \alpha=1$ and $m=10$ in (2), an optimal smoothing spline $x(t)\left(=x_{1}(t)\right)$ is computed based on the criterion (17) with $\Lambda\left(=\lambda_{1}\right)=0.001$ and $W_{i}=$ $1 / N$. Obviously, we impose the equality and inequality constraints

$$
\int_{t_{0}}^{t_{m}} x(t) d t=1, \quad x(t) \geq 0 \forall t \in\left[t_{0}, t_{m}\right],
$$

using the formulation described in Section 3.2. The results are shown in Figure 2, where the data points $\left(s_{i}, d_{i}\right)$ are shown by asterisks *, and the Gaussian probability density function $f(t)$ and the designed spline $x(t)$ are plotted in dash and solid lines respectively. Also we showed in dot line an optimal smoothing spline $x_{0}(t)$ obtained without the constraints (46). We see that the curve $x(t)$ closely approximates the Gaussian curve while maintaining the above constraint on density functions, which is not the case with the curve $x_{0}(t)$.

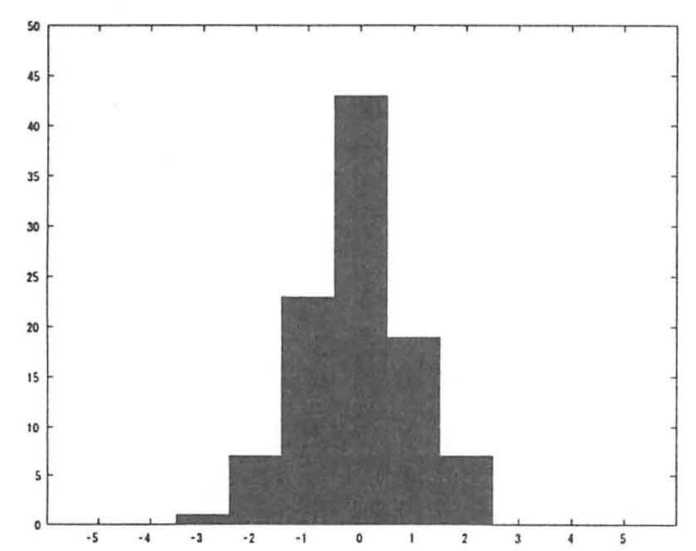

Fig. 1: Histogram of 100 Gaussian random numbers

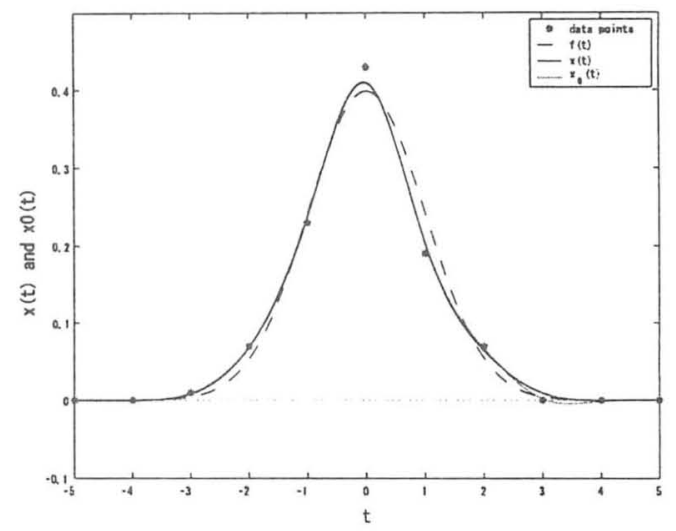

Fig. 2: Data points $\left({ }^{*}\right)$, constrained and unconstrained smoothing splines $\left(x(t)\right.$ and $x_{0}(t)$, resp.) for histogram from Gaussian probability density function $f(t)$.

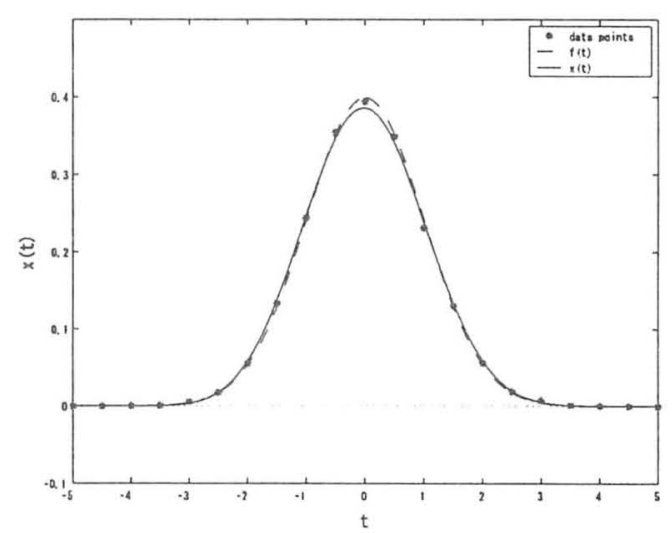

Fig. 3: Data points $\left({ }^{*}\right)$, constrained smoothing splines $x(t)$ for the case of 10000 samples, 21 bins $(N=21)$ and $m=20$ for histogram from Gaussian probability density function $f(t)$.

Obviously, we expect that, as the numbers of samples, bins and basis functions increase, the approximation improves. In fact we obtained the results as shown in Figure 3 for the case of 10000 random samples, $N=21$ and $m=20$.

\subsection{Approximation of Discontinuous Function}

For the case of $p=1$, we next tested the performance of constrained smoothing splines for approximating a discontinuous function $f(t)$ in $\left[t_{0}, t_{m}\right]=[-1,2]$, where $f(t)=1 t \in[0,1)$ and $f(t)=0$ otherwise. The data $\left(s_{i}, d_{i}\right), i=1,2, \cdots, N$ in (15) is generated by sampling $f(t)$ at $30(=N)$ points, $s_{i}$, equality spaced in $[-1,2]$, namely $d_{i}=f\left(s_{i}\right)$. The design parameters are $k=$ $3, \alpha=100 / 3$ and $m=100$ in (2), an optimal smoothing spline $x(t)$ is computed based on the criterion (17) with $\lambda=10^{-8}$ and $W_{i}=1 / N \forall i$. On designing the curve $x(t)$, we imposed the inequality constraints

$$
0 \leq x(t) \leq 1 \forall t \in\left[t_{0}, t_{m}\right]
$$




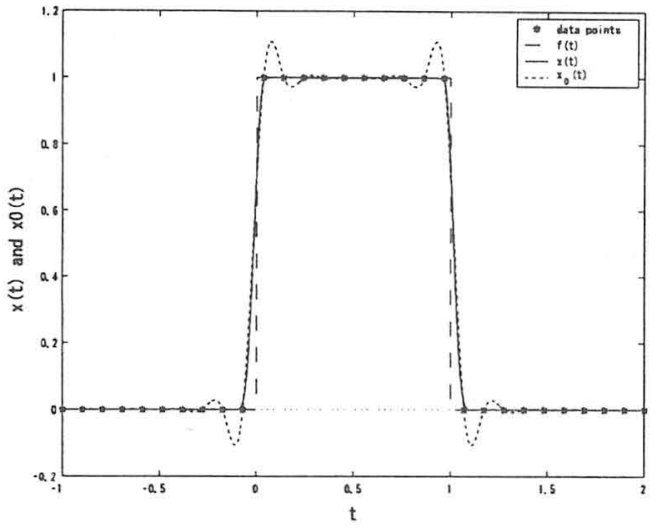

Fig. 4: Data points $\left(^{*}\right)$, constrained and unconstrained smoothing splines $\left(x(t)\right.$ and $x_{0}(t)$, resp.) for discontinuous function $f(t)$.

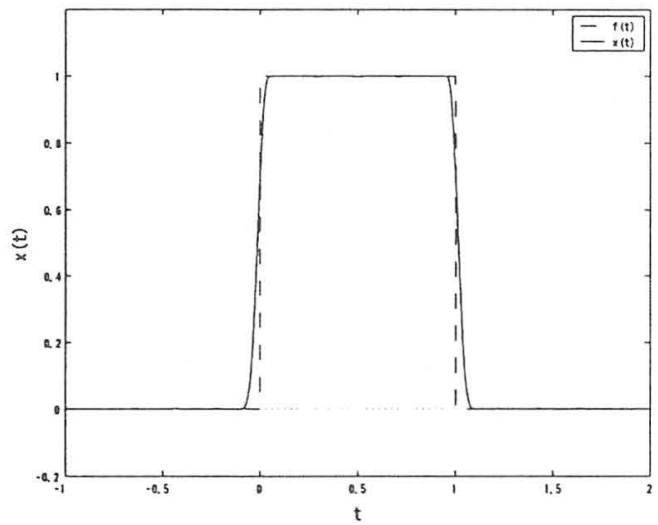

Fig. 5: Constrained smoothing spline $x(t)$ as approximation of $f(t)$.

The result is shown in Figure 4 (solid line) together with the true curve $f(t)$ (dash line), and a smoothing spline $x_{0}(t)$ (dot line) obtained without the constraints (47). Figure 5 depicts only $x(t)$ and $f(t)$ for the sake of clarity. Although it seems that the smoothing splines are not very suitable for approximating curve with jumps, we could recover the original function $f(t)$ surprisingly accurately. This is partly due to the design specifications that we used fairly many basis functions, i.e. $m=100$ for the interval $[-1,2]$ and that we used small smoothing parameter $\Lambda\left(=\lambda_{1}\right)=10^{-8}$. Gibbs-like phenomenon does not occur in $x(t)$ unlike the case of $x_{0}(t)$.

\subsection{Trajectory Planning}

We consider a trajectory planning problem with equality and inequality constraints for $p=2$. Specifically, we consider planning smoothing splines $x(t)=$ $\left[x_{1}(t) x_{2}(t)\right]^{T} \in \mathbf{R}^{2}$ in the time interval $\left[t_{0}, t_{m}\right]=[0,36]$ such that

$$
z^{-}(t) \leq x(t) \leq z^{+}(t), \forall t \in[0,36]
$$

where $z^{ \pm}(t) \in \mathbb{R}^{2}$ is $z^{ \pm}(t)=f(t) \pm \gamma$ for a function $f(t)=\left[f_{1}(t), f_{2}(t)\right]^{T} \in \mathbf{R}^{2}$ and a constant vector $\gamma \in$
$\mathbf{R}^{2}$. Here, we take the function $f_{i}(t)$ as

$$
f_{i}(t)=-\mathrm{e}^{a t} \cos (b t)+1, i=1,2
$$

with $a=\frac{1}{t_{m}} \log \frac{1}{4}$ and $b=\frac{6 \pi}{t_{m}}$, and $\gamma$ is set as $\gamma=0.2 \cdot 1_{2}$ with $\mathbb{1}_{i}=[1,1, \cdots, 1]^{T} \in \mathbb{R}^{i}$. We set $\alpha=1$ and $m=36$ in (2), hence the knot points are $t_{i}=i, i=$ $-3,-2, \cdots, m-1$. The initial and terminal conditions are set as

$$
\begin{aligned}
& x\left(t_{0}\right)=x^{(1)}\left(t_{0}\right)=x^{(2)}\left(t_{0}\right)=0_{2}, \\
& x\left(t_{m}\right)=0.75 \cdot \mathbf{1}_{2}, x^{(1)}\left(t_{m}\right)=x^{(2)}\left(t_{m}\right)=0_{2},
\end{aligned}
$$

and the magnitudes of the velocity and acceleration are limited at all the knot points as

$$
\left|x^{(1)}\left(t_{i}\right)\right| \leq 0.65 \cdot \mathbf{1}_{2},\left|x^{(2)}\left(t_{i}\right)\right| \leq 0.35 \cdot \mathbf{1}_{2},
$$

for $i=-3,-2, \cdots, m-1$.

Note that the inequality constraint in (66) is imposed by employing the limiting splines $x^{c \pm}(t)$ corresponding to $z^{ \pm}(t)$ as described in Section 3.2, i.e.

$$
x^{c-}(t) \leq x(t) \leq x^{c+}(t), \forall t \in[0,36] .
$$

In addition, (68) and (69) are pointwise constraints and the method in Section 3.1 can be used. For designing optimal smoothing splines $x(t)$, the data $d_{i} \in \mathbf{R}^{2}$ in (15) is obtained by sampling the curve $f(t)$. Here, the number of data is set as $N=15, s_{i}$ 's are randomly spaced in the interval $\left[t_{0}, t_{m}\right]=[0,36]$, and the magnitude of the additive Gaussian noise in $d_{i}$ is set as $\sigma=0.1$. The smoothing parameter $\Lambda$ and $W_{i}$ is set as $\Lambda=\operatorname{diag}\left\{10^{-3}, 10^{-3}\right\}$ and $W_{i}=I_{2}$ respectively. The optimal weight $\tau$ is computed together with the associated splines $x(t)$.

Figure 6 shows the results, $x_{1}(t)$ and its derivatives in solid lines, where the data points $\left(s_{i}, d_{i}\right)$ are shown by asterisk $*$. In the figure, the dot lines show the optimal splines $x_{1}^{0}(t)$ obtained without the constraints in (68), (69) and (70). The function $f_{1}(t)$ and limiting splines $x^{c \pm}(t)$ are plotted in dash and dotted-dash lines respectively. Also, the planned trajectory $x(t)$ in 3dimensional space $o-t, x_{1}, x_{2}$ is plotted in Figure 7 . We see that the trajectory $x(t)$ satisfies all the constraints. Note that the acceleration profile is piecewise linear due to cubic spline $(k=3)$, and hence the constraints in (70) at the knot points guarantees $\left|x^{(2)}(t)\right| \leq 0.35$. $\mathbf{1}_{2} \forall t \in[0,36]$. On the other hand, we see that the velocity constraint at the knot points yielded $\left|x^{(1)}(t)\right| \leq$ $0.65 \cdot 1_{2} \forall t \in[0,36]$ in this case, although it is not guaranteed theoretically.

\section{Concluding Remarks}

We developed a systematic method for designing optimal vector smoothing splines with equality and/or inequality constraints. The splines are constituted employing normalized uniform B-splines as the basis functions, and hence the central issue is to determine an 

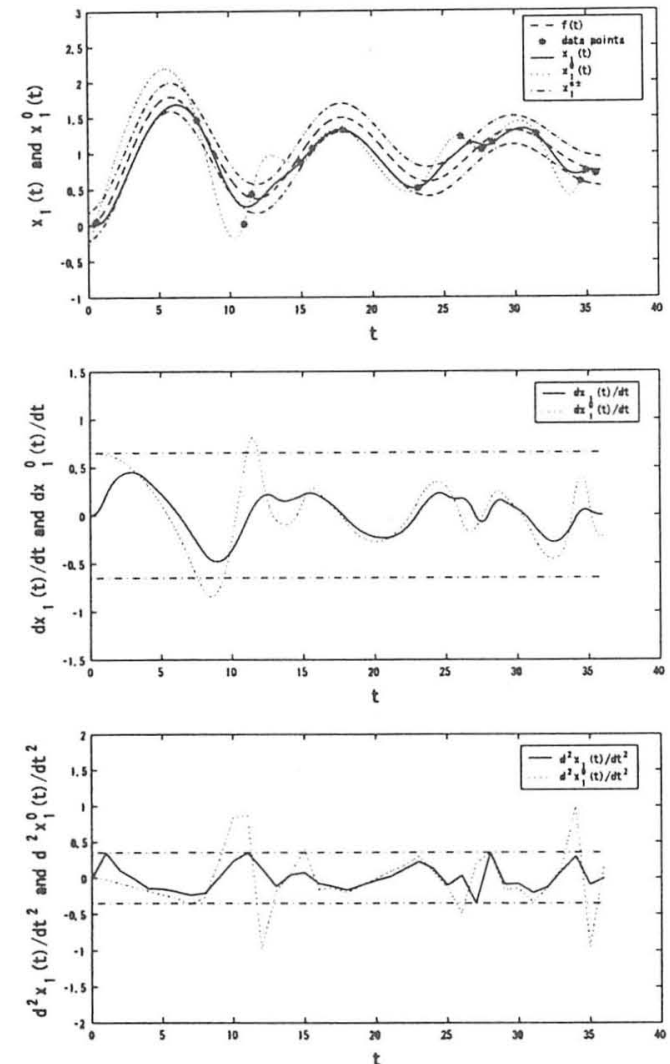

Fig. 6: Planned trajectories $x_{1}(t)$ (top), $\dot{x}_{1}(t)$ (middle) and $\ddot{x}_{1}(t)$ (bottom), and their counterparts $x_{1}^{0}(t), \dot{x}_{1}^{0}(t)$ and $\ddot{x}_{1}^{0}(t)$ without $(68),(69)$ and (70).

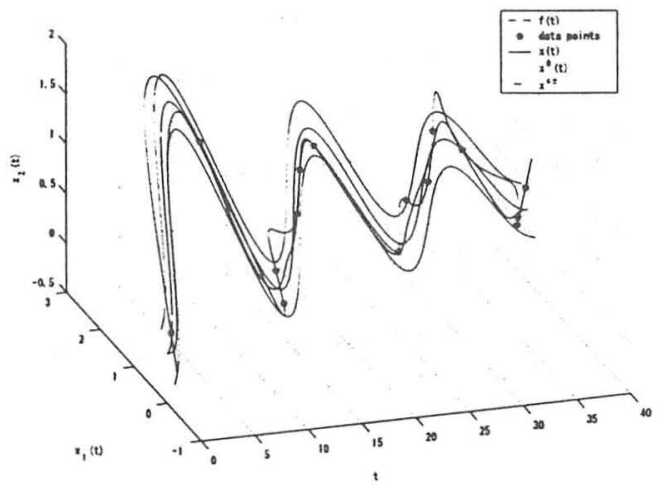

Fig. 7: Planned trajectory $x(t)$ plotted in 3-dimensional space $o-t, x_{1}, x_{2}$

optimal matrix $\tau$ of the so-called control points. Such an approach enables us to express various types of constraints as linear function of $\hat{\tau}(=\operatorname{vec} \tau)$, including those on the spline $x(t)$ and its elements, their derivatives and integral. The design problem becomes the quadratic programming problem in $\hat{\tau}$, where very efficient numerical algorithms are available. We examined the performances of the design method by numerical examples with equality and inequality constraints. To conclude, the developed method is effective as well as very useful for various types of problems.

\section{References}

[1] G. Wahba, Spline models for observational data, CBMS-NSF Regional Conference Series in Applied Mathematics, 59, Society for Industrial and Applied Mathematics (SIAM), Philadelphia, PA, 1990.

[2] Z. Zhang, J. Tomlinson, and C. F. Martin, Splines and linear control theory. Acta Appl. Math., 49, no. 1, 1-34, 1997.

[3] C. de Boor, A practical guide to splines, Revised Edition, Springer-Verlag, New York, 2001.

[4] H. Kano, H. Nakata, and C. F. Martin, Optimal Curve Fitting and Smoothing Using Normalized Uniform B-Splines : A tool for studying complex systems, Applied Mathematics and Computation, vol.169, no.1, pp.96-128, 2005.

[5] H. Fujioka, H. Kano, M. Egerstedt and C. Martin, Smoothing Spline Curves and Surfaces for Sampled Data, Int. J. of Innovative Computing, Information and Control, Vol.1, No.3, pp.429-449, 2005.

[6] M. Egerstedt and C. F. Martin, Optimal control and monotone smoothing splines. New trends in nonlinear dynamics and control, and their applications, 279-294, Lecture Notes in Control and Inform. Sci., 295, Springer, Berlin, 2003.

[7] C.F. Martin, S. Sun and M. Egerstedt, Optimal control, statistics and path planning. Computation and control, VI (Bozeman, MT, 1998). Math. Comput. Modelling, 33, no. 1-3, 237-253, 2001.

[8] H. Kano, H. Fujioka, and C. F. Martin, Optimal Smoothing and Interpolating Splines with Constraints, Proc. of the 46th IEEE Conference on Decision and Control, pp.3011-3016, New Orleans, LA, USA, Dec. 12-14, 2007.

[9] R. Dias, Density estimation via hybrid splines, $J$. of Statistical Computation and Simulation, Vol.60, Issue 4, pp.277-293, 1998.

[10] J. Z. Kolter and A. Y. Ng, Task-Space Trajectories via Cubic Spline Optimization, Proc. of the 2009 Int. Conf. on Robotics and Automation, pp.16751682, Kobe, Japan, May 12-17, 2009.

[11] P. Lancaster and M. Tismenetsky, The Theory of Matrices, Second Edition, Academic Press, 1985. 